\title{
Analysis of Low-Temperature Phase Diagram of the Microemulsion Model
}

\author{
E. I. Dinaburg and A. E. Mazel'
}

Shmidt Institute of Earth Physics, Academy of Sciences, USSR

Dedicated to Roland Dobrushin

\begin{abstract}
The low-temperature phase diagram of the microemulsion model is constructed for such values of its parameters when the model has only layered ground states.
\end{abstract}

\section{Introduction}

The microemulsion model is a lattice 3-dimensional spin model of statistical mechanics. It was proposed by Widom in [1] for the description of the microfilm structure in a mixture "oil-water." Also he showed that for some values of the mixture parameters the model can be reduced to a lattice spin model given by the Hamiltonian:

$$
\begin{aligned}
H\left(\varphi\left(\mathbb{Z}^{3}\right)\right)= & -I \sum_{\operatorname{dist}(x, y)=1} \varphi(x) \varphi(y)-J \sum_{\operatorname{dist}(x, y)=V^{2}} \varphi(x) \varphi(y) \\
& -K \sum_{\operatorname{dist}(x, y)=2} \varphi(x) \varphi(y),
\end{aligned}
$$

where $x, y \in \mathbb{Z}^{3}, \varphi\left(\mathbb{Z}^{3}\right)$ is a configuration on $\mathbb{Z}^{3}$ taking values in the set $\{ \pm 1\}$, $J=2 K$. We shall consider a more general model with any $I, J, K$. Clearly (see [2]) it is sufficient to consider only positive values of $I$.

First we investigate the ground states of the Hamiltonian (1.1). With this purpose we put

$$
O(x)=\left\{y \in \mathbb{Z}^{3}: \operatorname{dist}(x, y) \leqq 1\right\}
$$

and

$$
\begin{aligned}
U(\varphi(O(o))= & -0.5 I \sum_{\substack{\operatorname{dist}(x, y)=1 \\
x, y \in O(o)}} \varphi(x) \varphi(y)-0.5 J \sum_{\substack{\text { dist }(x, y)=\sqrt{2} \\
x, y \in O(o)}} \varphi(x) \varphi(y) \\
& -K \sum_{\substack{\text { dist }(x, y)=2 \\
x, y \in O(o)}} \varphi(x) \varphi(y) .
\end{aligned}
$$


Here $o$ is the origin of $\mathbb{Z}^{3}, \varphi(O(o))$ is the restriction of the configuration $\varphi\left(\mathbb{Z}^{3}\right)$ on the set $O(o)$. Then (1.1) can be rewritten as

$$
H\left(\varphi\left(\mathbb{Z}^{3}\right)\right)=\sum_{x \in \mathbb{Z}^{3}} U(\varphi(O(x))) .
$$

Further we restrict ourself by investigation of periodic ground states. Let $\psi\left(\mathbb{Z}^{3}\right)$ be a locally minimal configuration, i.e. such that

$$
U(\psi(O(x)))=\min U(\varphi(O(x))) .
$$

Then $\psi\left(\mathbb{Z}^{3}\right)$ is a ground state. Evidently any periodic ground state is the locally minimal configuration.

For any given triple $(I, J, K)$ of parameters we define the set $\Sigma(I, J, K)$ of configurations $\left\{\sigma_{i}(O(o))\right\}$ for which

$$
U\left(\sigma_{i}(O(o))\right)=\min _{\varphi(O(o))} U(\varphi(O(o)))=m(I, J, K) .
$$

As both the Hamiltonian (1.2) and the Hamiltonian (1.1) are invariant with respect to the \pm symmetry and permutations of coordinate axes the set $\Sigma(I, J, K)$ contains together with the configuration $\sigma$ the configurations $-\sigma, \sigma(S(O(o)))$, where $S$ is the coordinate axes permutation. If the configuration on $\mathbb{Z}^{3}$ can be "gathered" from configurations of $\Sigma(I, J, K)$ then this configuration is a ground state. In Fig. 1 we represent the diagram of ground states showing one element from every

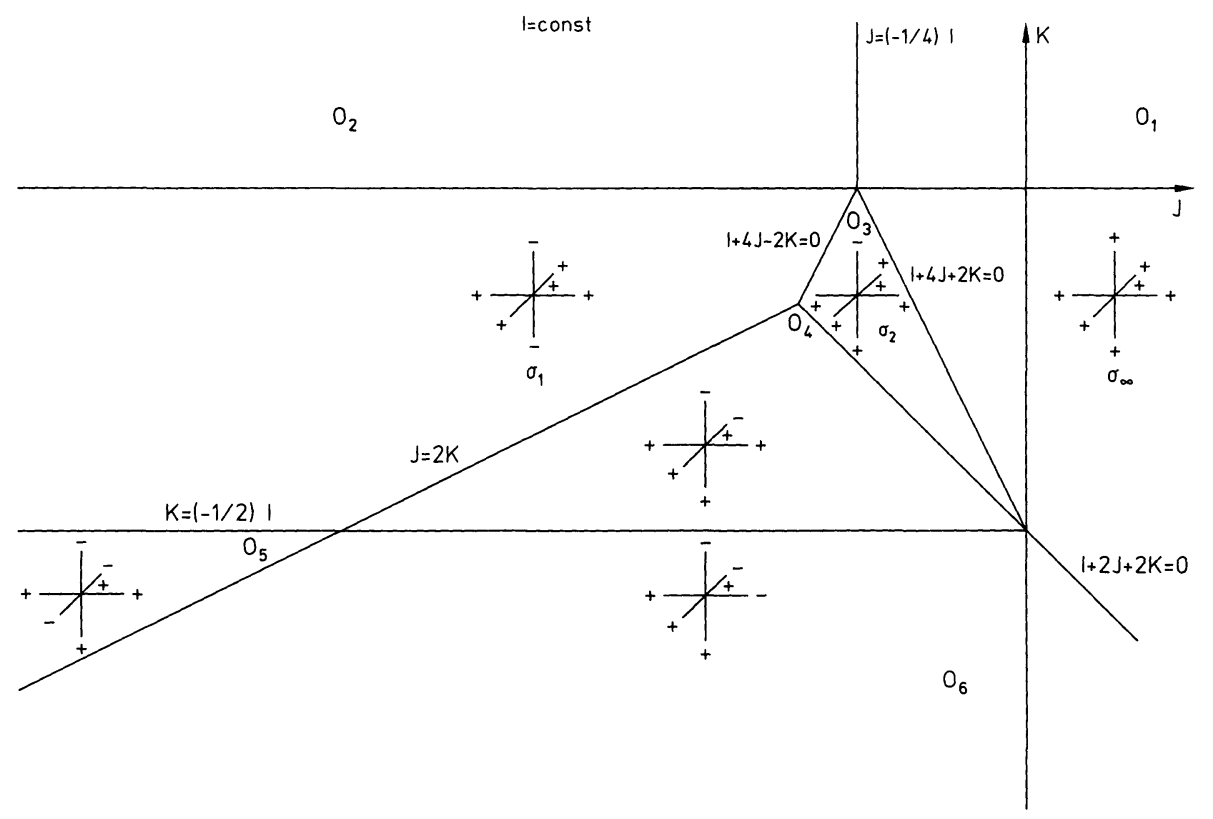

Fig. 1. Ground states of the model 
equivalence class of the set $\Sigma(I, J, K)$ for each pair $(J, K)$ when the parameter $I$ is fixed.

We shall consider only such parts of the diagram in which the all periodic ground states are layered configurations. Let us recall that a configuration is layered if it takes the constant value on any lattice plane orthogonal to one coordinate axis. Because of the described symmetries of the Hamiltonian it is sufficient to consider layered configurations which are constant on horizontal planes. According to the known notations of layered configurations (see [3]) a finite sequence $\left\langle l_{1}, \ldots, l_{k}\right\rangle$ of integer positive numbers defines the class of layered periodic configurations with the period $L$, where $L=\sum_{i=1}^{k} l_{i}$ if $k$ is even and $L=\sum_{i=1}^{k} 2 l_{i}$ if $k$ is odd. The class consists of an adjoining to each other of horizontal layers of thickness $l_{1}, l_{2}, \ldots, l_{k}$. A sequence of $l$ adjacent horizontal planes is called a layer of thickness $l$ of a configuration $\varphi$ if $\varphi$ takes the same constant value on these planes and the opposite value on the nearest planes to this sequence of planes. It is easy to see that such class contains precisely $2 \Sigma l_{i}$ configurations. There is a special notation $\langle\infty\rangle$ for the class consisting of two ferromagnetic configurations: $\varphi\left(\mathbb{Z}^{3}\right)$ $=+1, \varphi\left(\mathbb{Z}^{3}\right)=-1$.

In the domain $O_{1}$ there exist only the ferromagnetic ground states, in the domain $\mathrm{O}_{2}$ - only the class $\langle 1\rangle$, in the domain $\mathrm{O}_{3}$ - only class $\langle 2\rangle$. On the interior of the $\mathrm{O}_{2}$ and $\mathrm{O}_{3}$ common boundary there exists the infinite set of ground states consisting of any periodic layered configurations containing the layers of thickness 1 or 2 . At last on the interior of the interval on the line $I+4 J+2 K=0$ belonging to the boundary of domains $O_{1}$ and $O_{3}$ an arbitrary layered configuration is a ground state if it does not contain layers of thickness 1 . In the triple point $I=-4 J$, $K=0$ any layered configuration is a ground state.

For any given positive number $\varepsilon$ along the half-line $I=-4 J, K>\varepsilon$, there exists a finite number of periodic ground states and the Peierls condition is satisfied. Consequently in the neighbourhood of this half-line the low-temperature phase diagram can be investigated by the Pirogov-Sinai theory (see [4]).

In other cases both conditions necessary for the application of this theory are violated: a number of periodic ground states is infinite and the Peierls condition is not satisfied. Namely if the parameters $I, J, K$ belong to the line $I+4 J+2 K=0$ then the surface tension appears only along the vertical part of a phase-separation boundary; if $I, J, K$ belong to the line $I+4 J-2 K=0$, then the surface tension appears only along the edges of the phase-separation boundary.

For the phase diagram analysis we shall use some extension of the PirogovSinai theory applied to the study of phase transitions in the ANNNI model in [5] and stated in a general form in [6], where the so-called dominant ground states are considered instead of ground states. If the number of dominant ground states is finite and some additional conditions are valid, then as proved in [6] a theory analogous to the Pirogov-Sinai theory can be constructed.

The basic states of the theory are accounted in Sect. 2. Our conditions and terminology are somewhat different from the ones in [6]. But one can easily verify that for these conditions the theorem of Sect. 2 remains true. In Sect. 3 we find the dominant ground states of the model (1.1) and so we prove the following result. 
Theorem. For each fixed I and large enough inverse temperatures $\beta$ (I $\beta \gg 1)$ :

in a small neighbourhood of the triple point $J=-0.25 I, K=0$ there exists a full phase diagram (in the sense of [4] and [6]) for the phases $\langle 1\rangle,\langle 2\rangle,\langle\infty\rangle$ (see Fig. 2.a);

in a small neighbourhood of the interval $-I / 2<K<\beta^{-1}$ on the line $I+4 J$ $+2 K=0$ the finite number of phases from the set: $\langle 2\rangle,\langle 3\rangle,\langle 4\rangle,\langle\infty\rangle$ "survives" (see Fig. 2.c);

in a small neighbourhood of the interval $-I / 6<K<\beta^{-1}$ on the line $I+4 J$ $-2 K=0$ the finite number of phases from the set: $\langle 1\rangle,\langle 2\rangle,\langle 2,1\rangle$ "survives" (see Fig. 2.b).

Remark. In [1] and [2] it was surmised that the point $J=2 K$ on the line $I+4 J$ $+2 K=0$ is an ANNNI-like one, in particular, in its neighbourhood with finite $\beta$ an infinite number of phases exists. The theorem shows that this is not true.

a.

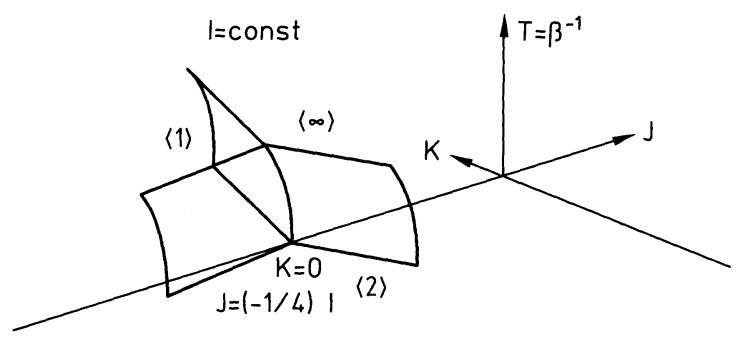

b.

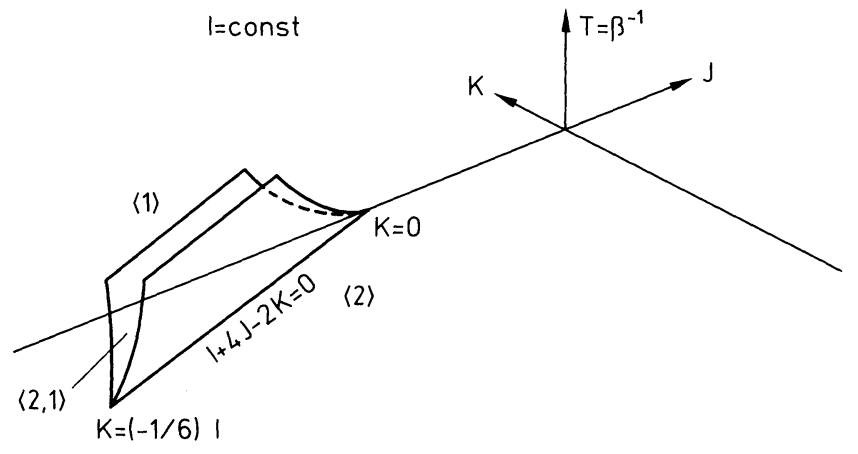

c.

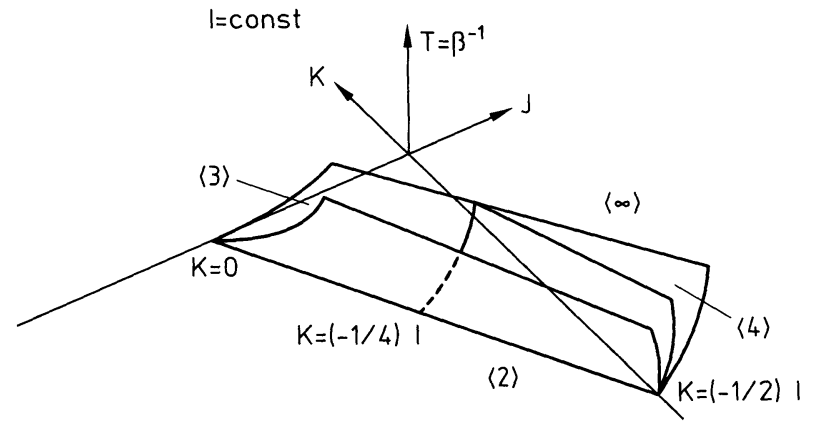

Fig. 2a-c. The phase diagram of the model 


\section{Description of the Method}

We consider lattice spin models on the integer-valued $v$-dimensional lattice $\mathbb{Z}^{v}$ with a finite set $\Phi$ of spin variable values defined by translation-invariant Hamiltonians $H$ with a finite interaction range. It is clear that the "value" of such a Hamiltonian on the configuration $\varphi\left(\mathbb{Z}^{v}\right)\left[\varphi(x) \in \Phi\right.$ for any $\left.x \in \mathbb{Z}^{v}\right]$ can be given as

$$
H\left(\varphi\left(\mathbb{Z}^{v}\right)\right)=\sum_{x \in \mathbb{Z}_{v}} U\left(\varphi\left(T_{x} E\right)\right),
$$

where $E$ is a finite set of the lattice containing the lattice origin $o, T_{x} E=E+x$ is the shift of set $E$ on the vector $x, U: \varphi(E) \rightarrow \mathbb{R}$ is a potential, $\varphi(E)$ is the restriction of $\varphi\left(\mathbb{Z}^{\nu}\right)$ on $E$. The set $T_{x} E$ is called an elementary volume (e.v.), restriction of $\varphi\left(\mathbb{Z}^{\nu}\right)$ on any e.v. is called an elementary configuration (e.c.). The e.c. $\sigma(E)$ is called the minimal one if $U(\sigma(E))=\min U(\varphi(E))=m$. The configuration $\varphi(V)$ in any volume $V$ with the boundary condition $\varphi(\bar{V})$ is a local ground state on $V$ if $\varphi\left(T_{x} E\right)$ is a minimal e.c. for any $x$ for which $T_{x} E \cap V \neq \emptyset$. (Here $\bar{V}$ is the complement of $V: \bar{V}=\mathbb{Z}^{v} \backslash V$.) It is evident the local ground state on $\mathbb{Z}^{v}$ is a ground state in a usual sense.

We suppose some conditions are valid for the Hamiltonian.

Condition 1. For the Hamiltonian (2.1) there exist at least one local ground state on $\mathbb{Z}^{\nu}$. If for some boundary condition $\varphi(\bar{V})(|V|<\infty)$ there exists its extension to the local ground state on $V$ then such an extension is unique.

Let $L>0$ be a constant. A set $A \in \mathbb{Z}^{v}$ is called $L$-connected if for any $a, b \in A$ there exists such a sequence of points $a_{0}, a_{1}, \ldots, a_{n}$ for which $a_{0}=a, a_{n}=b, a_{i} \in A$, and

$$
\operatorname{Dist}\left(a_{i}, a_{i+1}\right) \leqq L\left(\operatorname{Dist}(x, y)=\max _{1 \leqq j \leqq v}\left|x_{j}-y_{j}\right|, x=\left(x_{1}, \ldots, x_{v}\right), y=\left(y_{1}, \ldots, y_{v}\right)\right) .
$$

By definition a pair $\left(T_{x} E, \varphi\left(T_{x} E\right)\right)$ is an elementary defect if $U\left(\varphi\left(T_{x} E\right)\right) \neq m$, e.v. $T_{x} E$ is the support of the defect. A set of elementary defects is $L$-connected if the set of supports of these elementary defects is $L$-connected. Let $\varphi(V)$ be a configuration on $V$ and $\varphi(\bar{V})$ be a boundary condition. Then because of Condition 1 the configuration $\varphi(V)$ can be uniquely restored if the collection $\left\{\omega_{i}\right\}=\left\{\left(S_{i}, \varphi\left(S_{i}\right)\right)\right\}$ of connected components of elementary defects corresponding to it are given. For any such component $\omega_{i}$ put

$$
\Delta H\left(\omega_{i}\right)=\sum_{T_{x} E \cap S_{i} \neq \emptyset}\left[U\left(\varphi\left(T_{x} E\right)\right)-m\right],
$$

where $S$ is the support of the component.

Also put $R_{t}(A)=\left\{y \in \mathbb{Z}^{v}: \exists x \in A\right.$, Dist $\left.(x, y) \leqq t\right\}$ and

$$
R_{-t}(A)=\{x \in A: \operatorname{Dist}(x, \bar{A}) \geqq t\}
$$

for any $t>0$ and a set $A$.

Fix some constant $L>0$. The pair $\varepsilon=\left(S, \varphi\left(R_{L}(S)\right)\right)$ is called the perturbation of the local ground state $\psi(V)$ defined by boundary condition $\psi(\bar{V})$ if the following condition is valid: the perturbation support $S$ belongs to $V$ and is a $L$-connected subset of $\mathbb{Z}^{\nu} ; \varphi(x) \neq \psi(x)$ with $x \in S$ but $\varphi(x)=\psi(x)$ with $x \in R_{L}(S) \backslash S$. For every local 
ground state $\psi(V)$ the ensemble of perturbations compatible with this configuration is defined, i.e. such a collection $\left.\left\{\varepsilon_{i}\right\}=\left\{S_{i}, \varphi_{i}\left(R_{L}\left(S_{i}\right)\right)\right)\right\}$ for which

$$
\begin{gathered}
\varphi_{i}\left(R_{L}\left(S_{i}\right) \backslash S_{i}\right)=\psi\left(R_{L}\left(S_{i}\right) \backslash S_{i}\right), \\
R_{L}\left(S_{i_{1}}\right) \cap S_{i_{2}}=R_{L}\left(S_{i_{2}}\right) \cap S_{i_{1}}=\emptyset, \quad \varepsilon_{i_{1}}, \varepsilon_{i_{2}} \in\left\{\varepsilon_{i}\right\} .
\end{gathered}
$$

For any perturbation $\varepsilon_{i}$ its statistical weight

$$
W\left(\varepsilon_{i}\right)=\exp \left\{-\sum_{T_{x} E \cap S_{i} \neq \emptyset}\left[U\left(\varphi_{i}\left(T_{x} E\right)\right)-U\left(\psi\left(T_{x} E\right)\right)\right]\right\} .
$$

Further we also fix some constant $G>0$ called the main order.

Condition 2. Assume that the configuration $\varphi\left(\mathbb{Z}^{v}\right)$ is given and for some volume $V$ the configuration $\varphi(\bar{V})$ can be continued to the local ground state $\psi(V)$. Also assume that all connected components $\omega_{i}$ of elementary defects of $\varphi(V)$ satisfy the estimate $\Delta H\left(\omega_{i}\right) \leqq G$. Then there exists such $L=L(G)$ for which every element of the partition of the totality $\left\{\omega_{i}\right\}$ on L-connected components can be realized as the perturbation of $\psi(V)$.

From this point we shall use a special term "excitations" for perturbations which connected components of elementary defects $\omega_{i}$ satisfy the estimate: $\Delta H\left(\omega_{i}\right) \leqq G$.

Connected components of elementary defects $\omega$ with $\Delta H(\omega)>G$ are called clusters.

Clearly the Peierls condition: $w\left(\varepsilon_{i}\right)<\exp \left\{-\operatorname{const} \beta\left|S_{i}\right|\right\}$ holds for every excitation $\varepsilon_{i}$. It is well known that for large enough $\beta$ the satisfaction of this condition is sufficient for the diluteness of the excitation gas of the configuration $\psi(V)$, i.e. for the representation of the partition function logarithm

$$
Z(\psi(V), \beta \mid \psi(\bar{V}))=\sum_{\left\{\varepsilon_{i}\right\}} \prod_{i} w\left(\varepsilon_{i}\right)
$$

in the form of absolutely convergent series of polymers:

$$
\ln Z(\psi(V), \beta \mid \psi(\bar{V}))=\sum_{\pi \in V} q(\pi),
$$

where $\pi=\left(S, \varphi\left(R_{L}(S)\right)\right)$ is such a collection $\left\{\varepsilon_{j}\right\}$ of excitations compatible with $\psi(V)$ for which the set $S=\cup S_{j}$ is $L$-connected. Also $q(\pi)$ is uniquely defined by $\left\{\varepsilon_{j}\right\}$ and the estimation $|q(\pi)|<\exp \{-$ const $\beta|S|\}$ holds perhaps with another constant.

A configuration $\psi$ containing no excitations in the volume $V$ is called a factor configuration in this volume.

Fix the boundary condition $\psi(\bar{V})$ coinciding with some local ground state and consider the ensemble of factor configurations in $V$. On this ensemble define the factor model by the Hamiltonian $H_{G}$ :

$$
H_{G}(\psi(V))=\beta H(\psi(V))-H_{P}(\psi(V)),
$$

where $\psi(V)$ is the factor configuration in $V$,

$$
H_{P}(\psi(V))=\ln Z\left(\psi\left(V \backslash\left[\cup S_{i}\right]\right), \beta \mid \psi\left(\overline{V \backslash\left[\cup S_{i}\right]}\right)\right),
$$

$\cup S_{i}$ is the union of all cluster supports of the configuration $\psi(V)$. Here we include obviously the dependence on $\beta$ in the Hamiltonian $H_{G}$. 
One can see that

$$
\Xi_{V}(H, \beta \mid \varphi(\bar{V}))=\Xi_{V}\left(H_{G}\right),
$$

where in the left-hand side of this equality we have the partition function calculated by the Hamiltonian $H$ with boundary condition $\varphi(\bar{V})$ and for the inverse temperature $\beta$, but in the right-hand side we have the partition function of the factor model in volume $V$.

The factor configuration $\psi\left(\mathbb{Z}^{v}\right)$ is called the $G$-dominant ground state of the Hamiltonian $H_{G}$ if

$$
H_{G}\left(\psi\left(\mathbb{Z}^{v}\right)\right)-H_{G}\left(\psi_{1}\left(\mathbb{Z}^{v}\right)\right) \leqq 0
$$

for an arbitrary factor configuration $\psi_{1}\left(\mathbb{Z}^{\nu}\right)$ not coinciding with $\psi\left(\mathbb{Z}^{\nu}\right)$ only on a finite subset of $\mathbb{Z}^{\nu}$.

Let $c=\left\{C_{i}\right\}$ be a lattice partition on the cubes $C_{i}$ invariant with respect to shifts on periods of all $G$-dominant ground states.

Condition 3. Assume that only a finite number of periodic G-dominant ground states exists and that factor configuration $\psi$ coincides with neither G-dominant ground states on a finite set $A$ which is the union of cubes $C_{i}$. Then the inequality

$$
\left|H_{P}(\psi(A))-H_{P}(\kappa(A))\right|>\text { const } \exp (-\beta G)|A|
$$

is satisfied for any G-dominant ground state $\kappa$.

Theorem [6]. Let the Hamiltonian $H$ satisfy Conditions 1 and 2. Consider the perturbated Hamiltonian $H=H_{0}+\sum_{i=1}^{n-1} \mu_{i} H_{i}$. Suppose that the corresponding factor model has on a curve $\mu=\mu_{0}(\beta)\left(\beta>\beta_{0}\right)$ precisely $n$ G-dominant ground states satisfying Condition 3 and assume that the Hamiltonians $H_{0}, \ldots, H_{n-1}$ remove the degeneracy of the G-dominant ground states. Then for sufficiently large inverse temperatures $\beta>\beta_{0}$ and for some constant $C>0$ there exists a full phase diagram in the domain

Here $\mu=\left(\mu_{1}, \ldots, \mu_{n-1}\right)$.

$$
\left|\mu-\mu_{0}(\beta)\right|<\exp \{-\beta C G\} .
$$

The theorem has an evident extension on the case when all $H_{i}(i=0,1, \ldots, n-1)$ have a symmetry group $\Gamma$. Then the theorem is true for classes of $\Gamma$-equivalent $G$-dominant ground states.

\section{Dominant Ground States of the Microemulsion Model}

We investigate the domains defined by the following constraints:

1.

$$
I \beta \gg 1 ;|K|,|I+4 J|<\beta^{-1} \exp \{-7 \beta I\},
$$

2. $I \beta \gg 1 ;-J \beta \gg 1 ;-I / 2<K<-\beta^{-1} ; \beta|I+4 J+2 K|<\exp \{-\beta\}$,

3.

$$
I \beta \gg 1 ;-I / 6<K<-\beta^{-1} ; \beta|I+4 J-2 K|<\exp \{-\beta\} .
$$

To verify Condition 1 let us consider the finite volume $V$ with the boundary condition $\varphi(\bar{V})$ and for every horizontal plane $\mathbb{Z}^{2} \subset \mathbb{Z}^{3}$ intersecting $V$ denote connected components of $\mathbb{Z}^{2} \cap V$ by $\mathbb{Z}_{i}^{2}$ and their boundary $\mathbb{Z}^{2} \cap\left[R_{1}\left(\mathbb{Z}_{i}^{2}\right) \backslash \mathbb{Z}_{i}^{2}\right]$ by 
$\partial \mathbb{Z}_{i}^{2}$. Obviously $\varphi(\bar{V})$ can't be continued to the layered configuration $\varphi(V)$ if $\varphi\left(\partial \mathbb{Z}_{i}^{2}\right)$ $\neq$ const, but for every $\mathbb{Z}_{i}^{2}$ the unique continuation $\varphi\left(\mathbb{Z}_{i}^{2}\right)=a$ exists if $\varphi\left(\partial \mathbb{Z}_{i}^{2}\right)=a$, i.e. Condition 1 is valid.

To verify Condition 2 consider the configuration $\varphi\left(\mathbb{Z}^{3}\right)$ with all connected components of elementary defects $\omega_{i}=\left(S_{i}, \varphi\left(S_{i}\right)\right)$ belonging to the finite volume $V$, satisfying the estimation $\Delta H\left(\omega_{i}\right) \leqq G$, and hence the estimation $\left|S_{i}\right|<M$ with constant $M=M(G)$. Suppose the volume $V$ is so large that configuration $\varphi(\bar{V})$ has continuation to the local ground state $\psi(V)$. Consider $\varphi(V)$ as the collection $\left\{S_{j}\right\}$ of 2-connected sets on which $\varphi(V) \neq \psi(V)$. Imagine $S_{j}$ as the union of belonging $\mathbb{R}^{3}$ closed unit cubes centered at $x \in S_{j}$ and notice that in the configuration $\varphi(V)$ its elementary defects are placed at least along all edges of the polyhedron obtained. Consequently for every connected component of this polyhedron's edges the total edge length is smaller than $M$, the set of all polyhedron edges is $M^{2}$-connected and so the set corresponding to the $S_{j}$ connected components of elementary defects is $M^{2}$-connected.

As Conditions 1 and 2 are valid with an arbitrary $G$ and as the number of polymers with the statistical weight which is not $o(\exp \{-\beta G\})$ is finite with respect to $\mathbb{Z}^{3}$ translations it is convenient to check Condition 3 according to the following scheme. First all polymers with the statistical weight $\exp \left\{-\beta G_{1}\right\}$ which is maximal for given values of parameters are looked for and $G_{1}$-dominant ground states are calculated. If the number of these states appears to be infinite then again polymers with the statistical weight $\exp \left\{-\beta G_{2}\right\}$ which is maximal among the remaining ones are looked for and $G_{2}$-dominant ground states are calculated. The procedure repeats until for some $G_{n}$ the number of $G_{n}$-dominant ground states becomes finite. After that the estimation (2.5) is verified and in case of its validity $G_{n}$ may be selected as the final main order.

Obviously for calculating of $G$-dominant ground states satisfying this estimation the truncated polymer Hamiltonian $H_{P}^{T}$ obtained from the full Hamiltonian $H_{P}$ by cutting all terms that are $o(\exp \{-\beta G\})$ can be used. As the polymer $\pi$ statistical weight $q(\pi)$ satisfies the Peierls condition, the Hamiltonian $H_{P}^{T}$ has the finite interaction radius and a Hamiltonian $H_{G}^{T}=\beta H-H_{P}^{T}$ can be written in the form:

$$
\begin{aligned}
H_{G}^{T}\left(\psi\left(\mathbb{Z}^{3}\right)\right)= & \sum_{\mathbb{Z} \subset \mathbb{Z}^{3}}\left[\sum_{A \cong E}|A \cap \mathbb{Z}||A|^{-1} U(\psi(A))\right. \\
& \left.-\sum_{\pi=\left(S, \varphi\left(R_{L}(S)\right)\right)}|S \cap \mathbb{Z}||S|^{-1} q(\pi)\right],
\end{aligned}
$$

where external summation goes over all vertical lines $\mathbb{Z} \subset \mathbb{Z}^{3}$. In the class of layered configurations the Hamiltonian $H_{G}^{T}$ is invariant with respect to horizontal shifts, hence in (3.4) all terms of the external sum are equivalent and represent the onedimensional Hamiltonian $\widetilde{H}_{G}(\psi(\mathbb{Z}))$.

Each term which appears in the $\widetilde{H}_{G}$ naturally corresponds to the sequence $\sigma$ of + and - which is either the projection of $\psi(A)$ on $\mathbb{Z}$ or the projection of $\psi\left(R_{L}(S) \backslash S\right)$ on $\mathbb{Z}$. The sum over all such terms which are corresponding to the fixed $\sigma$ defines the potential $\tilde{U}_{\sigma}$. Expressed by $\tilde{U}_{\sigma}$ the specific energy of the periodic configuration $\varphi(\mathbb{Z})$ is equal to

$$
h(\varphi)=\sum_{\sigma} \tilde{U}_{\sigma} n_{\sigma}(\varphi),
$$

where $n_{\sigma}(\varphi)$ is the occurrence frequency of the sequence $\sigma$ in the configuration $\varphi$. 
In general frequencies $n_{\sigma}(\varphi)$ are linearly dependent. Suppose that the representation of certain frequencies by other ones is known:

$$
n_{\sigma_{i}}=\sum_{i} a_{i j} n_{\sigma_{j}}
$$

Then for $\sigma_{i}$ we can define new potentials

$$
U_{\sigma_{i}}=\tilde{U}_{\sigma_{i}}+\sum_{j} a_{i j} \tilde{U}_{\sigma_{j}}
$$

and can rewrite expression (3.5) as

$$
h(\varphi)=\sum_{i} U_{\sigma_{\imath}} n_{\sigma_{i}}(\varphi) .
$$

In the rest of our paper we shall often use such transformations and the fact that if $\sum_{i} n_{\sigma_{i}} C_{i}=1$ for all $\varphi$ from some configuration class, then in the space of model parameters the system of equations

$$
C_{i}^{-1} U_{\sigma_{i}}=\text { const }
$$

defines the hypersurface on which specific energies coincide for all $\varphi$ from this class.

Let us pass over to the concrete calculations. The domain (3.1) in the space $(I, J, K, \beta)$ is the small neighbourhood of the hypersurface $I+4 J=0, K=0$ which minimal elementary configurations $\sigma_{1}, \sigma_{2}, \sigma_{\infty}$ (see Fig. 1) coexist on, and thus all layered configurations are ground states. In this neighbourhood it is convenient to use the coordinates $\delta=I+4 J+2 K, \mu=I+4 J-2 K$ and to construct the phase diagram in the space $(\delta, \mu, \beta)$.

Potentials of the minimal elementary configurations are equal to

$$
\begin{gathered}
U\left(\sigma_{\infty}\right)=1.5 I+1.5 \delta ; \quad U\left(\sigma_{2}\right)=1.5 I+0.5 \delta ; \\
U\left(\sigma_{1}\right)=1.5 I+0.5 \delta-\mu,
\end{gathered}
$$

and the statistical weight of an arbitrary excitation has the form:

$$
W(\varepsilon)=\exp \{-l \beta I-m \beta \delta-n \beta \mu\}, \quad l>0, m, n \in \mathbb{R} .
$$

Using the closeness of $\beta \delta$ and $\beta \mu$ to zero in the further calculations we shall first take into account only the main terms of power series for $\exp \{-m \beta \delta\}$ and $\exp \{-n \beta \mu\}$, i.e. we shall consider $W(\varepsilon)=\left.W(\varepsilon)\right|_{\delta=\mu=0}$. Then we are to correct the results obtained taking into account the full power series.

In general the statistical weight of the excitation $\varepsilon=\left(S, \varphi\left(R_{L}(S)\right)\right)$ depends on the whole configuration $\varphi\left(R_{L}(S)\right)$, but with $\delta=\mu=0$ it is defined only by the value $\varphi$ on $S$ since in this case the single-site excitation energy is clearly equal to $6 \beta I$ (see Fig. 3a) and the multi-site excitation energy is equal to the sum of energies of corresponding single-site excitations minus doubled interaction energies of unit bonds, diagonal planar bonds and double bonds belonging to the support of $\varepsilon$.

All polymers with the statistical weight $\geqq \exp \{-11 \beta I\}$ are enumerated in Fig. $3 b$ in decreasing weight order. Since their diameters $\leqq 3$ for any periodic configuration $\varphi(\mathbb{Z})$ the specific energy $h(\varphi)$ corresponding to the Hamiltonian $H_{11 I}$ can be calculated by the formula

$$
h(\varphi)=\sum_{\sigma \in\{+++,++-,-++,+-+, \ldots,--+,+-,,++\}} n_{\sigma}(\varphi) U_{\sigma},
$$


a.

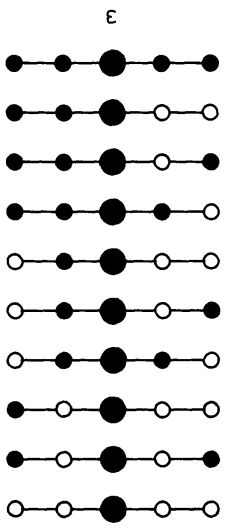

$(-1 / \beta) \ln w(\varepsilon)$

$121+24 J+12 K$

$81+8 J+8 K$

$8 \mathrm{l}+8 \mathrm{~J}+12 \mathrm{~K}$

$121+24 J+8 K$

$81+8 J+4 K$

$81+8 \mathrm{~J}+8 \mathrm{~K}$

$121+24 J+4 K$

$41-8 J+8 K$

$4 k-8 J+12 K$

$4 I-8 J+4 K$

vertikal

b.

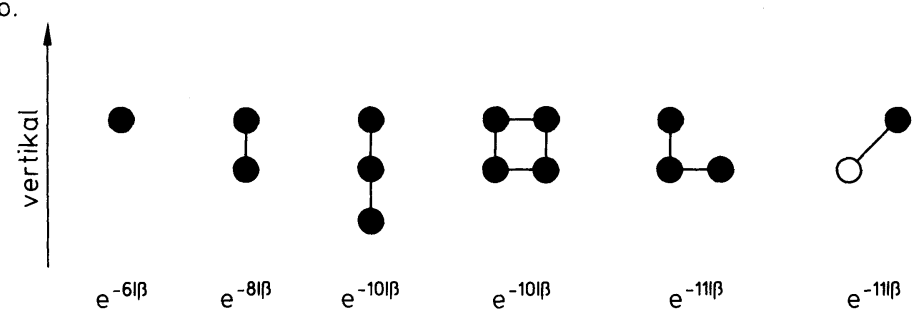

c.

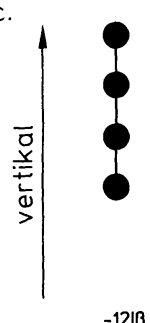

d.

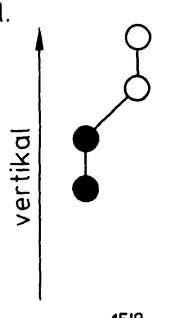

e.

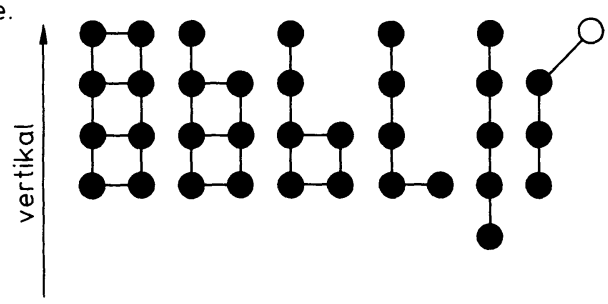

Fig. 3a-c. Excitations and their statistical weights near the triple point (Notations: black and white circles correspond to spins of opposite signs. Bigger circles show the sites where spin-flips have to be done)

where $U_{\sigma}$ are defined with the help of identities:

$$
\begin{gathered}
n_{+}=n_{+++}+(2 / 3)\left[n_{+-+}+n_{++-}+n_{-++}\right] \\
+(1 / 3)\left[n_{+-+}+n_{+--}+n_{-++}\right], \\
n_{++}=n_{+++}+(1 / 2)\left[n_{++-}+n_{-++}\right], \\
n_{+-}=(1 / 2)\left[n_{+-+}+n_{-+-}+n_{++-}+n_{-++}\right],
\end{gathered}
$$

and analogous identities for $n_{-}, n_{--}, n_{-+}$. 
Clearly $\sum n_{\sigma}(\varphi)=1$ and $U_{\sigma}=U_{-\sigma}$, therefore in the space $(\delta, \mu, \beta)$ the system of equations

$$
U_{+++}=U_{++-}=U_{+-+}
$$

defines the coexistence curve of $11 I$-dominant ground states constructed from minimal elementary configurations $\sigma_{1}, \sigma_{2}, \sigma_{\infty}$ and systems

$$
\begin{aligned}
& U_{+++}<U_{++-}=U_{+-+}, \\
& U_{++-}<U_{+++}=U_{+-+}, \\
& U_{+-+}<U_{+++}=U_{++-}
\end{aligned}
$$

define coexistence surfaces of $11 I$-dominant ground states constructed from minimal elementary configurations $\sigma_{1}$ and $\sigma_{2}, \sigma_{1}$ and $\sigma_{\infty}, \sigma_{2}$ and $\sigma_{\infty}$ respectively. After elementary calculations one can find the solutions of the system (3.15):

$$
\begin{gathered}
\delta=-\beta^{-1}(0.5 \exp \{-8 \beta I\}+2 \exp \{-10 \beta I\}+2.5 \exp \{-11 \beta I\}), \\
\mu=-\beta^{-1}(0.5 \exp \{-8 \beta I\}+\exp \{-10 \beta I\}-1.5 \exp \{-11 \beta I\} .
\end{gathered}
$$

Solutions of systems (3.16), (3.17), (3.18) can be found in an analogous way.

Obviously as the main order increases no polymers with supports a height $\leqq 3$ can remove an infinite degeneracy of the dominant ground state on hypersurfaces defined by systems (3.15-3.18). Such polymers only add terms of a corresponding order to the right-hand sides of (3.19-3.20).

The excitation shown in Fig. $3 \mathrm{c}$ has the maximal statistical weight among polymers with height $\geqq 4$. This excitation corresponds to the sequences ++++ and ---- , therefore taking into account that excitation the specific energy $h(\varphi)$ is equal to

$$
\begin{aligned}
h(\varphi)= & \sum_{\sigma \in\{+++,++-,-++,+-+, \ldots,--+,+--,-+-\}} n_{\sigma}(\varphi) U_{\sigma} \\
& -\left[n_{++++}(\varphi)+n_{----}(\varphi)\right] \exp \{-12 \beta I\} .
\end{aligned}
$$

Hence the equation $h(\langle 2\rangle)=h(\langle\infty\rangle)$ is transformed to the

$$
U_{++-}=U_{+++}-\exp \{-12 \beta I\} \text {. }
$$

The configuration consisting of layers of thickness $m \geqq 3$ can't already be $12 I$ dominant ground states on the surface defined by this equation because

$$
n_{++++}(\langle m\rangle)+n_{-} \ldots(\langle m\rangle)=(m-3) / m
$$

and

$$
\begin{aligned}
h(\langle m\rangle) & =\left(U_{-++}+(m-2) U_{+++}+U_{++-}\right) / m-\exp \{-12 \beta I\}(m-3) / m \\
& =h(\langle\infty\rangle)+\exp \{-12 \beta I\} / m>h(\langle\infty\rangle) .
\end{aligned}
$$

Thus the excitation which we have considered removes the infinite degeneracy of $11 I$-dominant ground states on the coexistence surface of configurations $\langle 2\rangle$ and $\langle\infty\rangle$, but clearly one can't remove a similar degeneracy on the coexistence surface of configurations $\langle 1\rangle$ and $\langle 2\rangle$. 

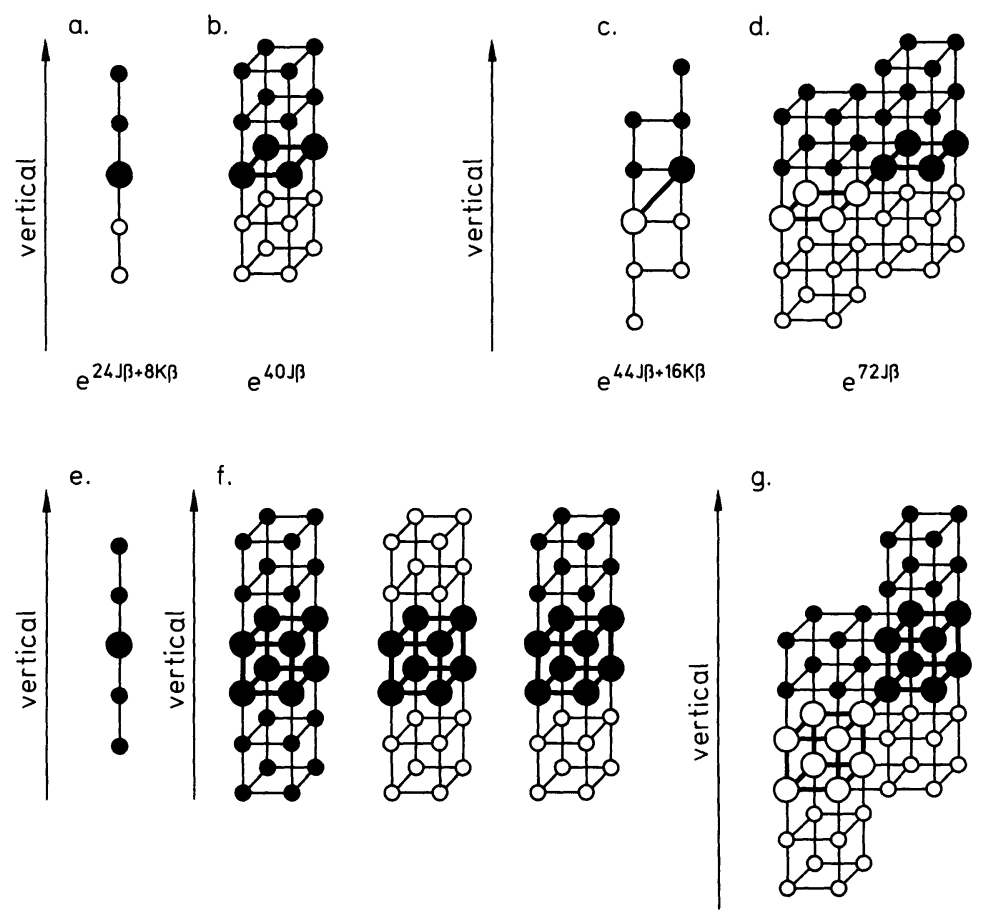

$e^{24 / \beta+12 K \beta}$

$e^{48 . j \beta}$

$\mathrm{e}^{88 \mathrm{~J} / \mathrm{\beta}}$

Fig. 4a-d. Excitations and their statistical weights near the line $I+4 J+2 K=0$

The excitation removing this degeneracy arises only in the order $15 I$ and is shown in Fig. 3d. Taking into account the given excitation for configurations $\varphi$ consisting only of layers of thickness 1 or 2 we have

$$
\begin{aligned}
h(\varphi)= & \sum_{\sigma \in\{++-,-++,+-+,--+,+--,-+-\}} n_{\sigma}(\varphi) U_{\sigma} \\
& -\left[n_{++--}(\varphi)+n_{--++}(\varphi)\right] \exp \{-15 \beta I\},
\end{aligned}
$$

and on the surface defined by the equation $h(\langle 1\rangle)=h(\langle 2\rangle)$ a minimum of $h(\varphi)$ is reached only on configurations $\langle 1\rangle$ and $\langle 2\rangle$. Other polymers with the statistical weights

$$
\exp \{-12 \beta I\}<q(\pi)<\exp \{-15 \beta I\}
$$

either have height $\leqq 3$ or (see Fig. 3e) only strengthen the inequality (3.22).

Thus in the order $15 I$ there is a full phase diagram for phases $\langle 1\rangle,\langle 2\rangle,\langle\infty\rangle$ in the small neighbourhood of the curve $\delta=\delta(\beta), \mu=\mu(\beta)$, defined by equations $h(\langle 1\rangle)$ $=h(\langle 2\rangle)=h(\langle\infty\rangle)$.

Finally we must correct our results taking into account true polymers statistical weights. Since $\beta \delta$ and $\beta \mu$ obtained are $O(\exp \{-8 \beta I\})$ only two terms: $\left.m \beta \delta W(\varepsilon)\right|_{\delta=\mu=0}$ and $\left.n \beta \mu W(\varepsilon)\right|_{\delta=\mu=0}$ are not $o(\exp \{-15 \beta I\})$ in the power series for $W(\varepsilon)$. Furthermore they are not $o(\exp \{-15 \beta I\})$ only for $\left.W(\varepsilon)\right|_{\delta=\mu=0} \leqq \exp \{-7 \beta I\}$. The preceding condition is valid only for single-site excitations and it is easy to 
verify that such additional terms can't influence the fullness of the phase diagram obtained.

The domain (3.2) in the space $(I, J, K, \beta)$ is the small neighbourhood of the hypersurface $I+4 J+2 K=0$ which minimal elementary configurations $\sigma_{1}$ and $\sigma_{\infty}$ (see Fig. 1) coexist on and thus all layered configurations containing no layers of thickness 1 are ground states. In this neighbourhood it is convenient to use the coordinate $\delta=I+4 J+2 K$ and to construct the phase diagram in the space $(\delta, \beta)$.

It can be verified that with $K \geqq 2 J$ the excitation shown in Fig. 4a and with $K \leqq 2 J$ the excitation shown in Fig. 4 b have the maximal statistical weight among the weights of all polymers. Let $G_{1}=\min \{-24 J-8 K,-40 J\}$. In the order $G_{1}$ the specific energy of the periodic configuration $\varphi$ containing no layers of thickness 1 is equal to

$$
\begin{aligned}
h(\varphi)= & \sum_{\sigma \in\{++-,-++,+++,--+,+--,---\}} n_{\sigma}(\varphi) U_{\sigma} \\
& -\sum_{\sigma \in\{+++-,,--+++,--+++,++---\}} n_{\sigma}(\varphi) \exp \left\{-\beta G_{1}\right\},
\end{aligned}
$$

where $U_{\sigma}$ are defined by means of identities (3.12-3.14). In the expression (3.23) the first sum does not depend on $\varphi$ and the second sum is maximal when $\varphi=\langle 3\rangle$. Therefore in defining the curve $\delta_{1}(\beta)$ as the solution of the equation $h(\langle 3\rangle)=h(\langle 2\rangle)$ and curve $\delta_{2}(\beta)$ as the solution of the equation $h(\langle 3\rangle)=h(\langle\infty\rangle)$ we have that with $\delta \leqq \delta_{1}$ configurations $\langle 2\rangle$ are the $G_{1}$-dominant ground states, with $\delta_{1} \leqq \delta \leqq \delta_{2}$ configurations $\langle 3\rangle$ are the $G_{1}$-dominant ground states, with $\delta \geqq \delta_{2}$ configurations $\langle\infty\rangle$ are the $G_{1}$-dominant ground states.

The specific energy of configuration $\varphi$ consisting only of layers of thickness 2 or 3 can be calculated as

$$
h(\varphi)=\sum_{\sigma \in\{+--+,-++-\}} n_{\sigma}(\varphi) U_{\sigma}+\sum_{\sigma \in\{+++,---\}} n_{\sigma}(\varphi) U_{\sigma},
$$

where $U_{\sigma}$ are obtained with the help of identities:

$$
\begin{gathered}
n_{+}=3 n_{+++}+2 n_{-++-}, \\
n_{++}=n_{-++-}+2 n_{+++}, \\
n_{+++--}=n_{+++},
\end{gathered}
$$

and analogous identities for $n_{-}, n_{--}, n_{--+++}$. Besides

$$
\sum_{\sigma \in\{+--+,-++-\}} 2 n_{\sigma}(\varphi)+\sum_{\sigma \in\{+++,---\}} 3 n_{\sigma}(\varphi)=1 .
$$

Therefore all similar configurations are $G_{1}$-dominant ground states on the curve $\delta_{1}(\beta)$ and no polymers with supports belonging either to the layer of thickness 2 or to the layer of thickness 3 can remove this infinite degeneracy. Among polymers intersecting the boundary between layers with $K>1.75 \mathrm{~J}$ the excitation illustrated in Fig. 4c and with $K<1.75 \mathrm{~J}$ the excitation illustrated in Fig. $4 \mathrm{~d}$ have the maximal statistical weight. These excitations correspond to the sequences +++--and ---+++ and consequently remove the infinite degeneracy of the $G_{1}$-dominant ground state because in the order $G_{2}=\min \{-44 J-16 \mathrm{~K},-72 J\}$ the maximum of the $n_{+++---}(\varphi)+n_{--++++}(\varphi)$ is achieved on the configuration $\langle 3\rangle$. 
On the curve $\delta_{2}(\beta)$ specific energies of the configurations which do not contain layers of thickness 1 and 2 coincide because with $U_{\sigma}$ calculated by means of identities:

$$
n_{++-}=n_{+--}=n_{+++-}=n_{++--}, \quad n_{-++}=n_{--+}=n_{---++}=n_{--++}
$$

the expression for $h(\varphi)$ takes the form:

$$
h(\varphi)=\sum_{\sigma \in\{++--,--++\}} n_{\sigma}(\varphi) U_{\sigma}+\sum_{\sigma \in\{+++,--\}} n_{\sigma}(\varphi) U_{\sigma},
$$

and

$$
\sum_{\sigma \in\{++-,,--++\}} 2 n_{\sigma}(\varphi)+\sum_{\sigma \in\{+++,---\}} n_{\sigma}(\varphi)=1 .
$$

With $K \geqq 2 J$ the excitation introduced in Fig. 4e has the maximal statistical weight among polymers that do not coincide with polymers shown in Fig. 4a and b. Since this excitation corresponds to the sequences +++++ and ---for $m \geqq 4$

$$
\begin{aligned}
h(\langle m\rangle) & =U_{+++}(m-2) / 2+U_{++--} / m-\exp \{24 \beta J+12 \beta K\} / m \\
& =h(\langle\infty\rangle)+\exp \{24 \beta J+12 \beta K\} / m
\end{aligned}
$$

on the curve defined by the equation

$$
h(\langle 3\rangle)=h(\langle\infty\rangle)\left(\langle=\rangle U_{++--}=2 U_{+++}-3 \exp \{24 \beta J+12 \beta K\}\right) .
$$

Hence infinite degeneracy of the dominant ground state is removed in the order $-24 \beta J-12 \beta K$.

With $K \leqq 2 J$ the statistical weight of the excitation shown in Fig. 4e becomes smaller than the statistical weight of the excitation shown in Fig. $4 \mathrm{f}$ and corresponding to the sequences: $++++++,-\ldots-\ldots,++---$, --++++ . Because of this excitation the curve $\delta_{2}(\beta)$ is splitting and phase $\langle 4\rangle$ arises between phases $\langle 3\rangle$ and $\langle\infty\rangle$. Moreover there is no other $-48 \mathrm{~J}$-dominant ground states on the coexistence curve of phases $\langle 4\rangle$ and $\langle\infty\rangle$, but on the coexistence curve of phases $\langle 3\rangle$ and $\langle 4\rangle$ all configurations containing only layers of thickness 3 and 4 are $-48 J$-dominant ground states.

This infinite degeneracy is removed in the order $-88 \mathrm{~J}$ by means of the excitation shown in Fig. 4g.

It is easy to verify that if $K=2 J$, then only the configurations $\langle 3\rangle,\langle 4\rangle,\langle\infty\rangle$ are $-88 \mathrm{~J}$-dominant ground states.

The domain (3.3) in the space $(I, J, K, \beta)$ is the small neighbourhood of the hypersurface $I+4 J-2 K=0$ which minimal elementary configurations $\sigma_{1}$ and $\sigma_{2}$ (see Fig. 1) coexist on and thus all layered configurations consisting of layers of thickness 1 and 2 are ground states. In this neighbourhood it is convenient to use the coordinate $\mu=I+4 J-2 K$ and to construct the phase diagram in the space $(\mu, \beta)$.

Investigation of this region can be carried out analogously to the investigation of the regions mentioned above therefore we only indicate excitations removing the infinite degeneracy of the dominant ground states. With $K \geqq-0.1 I$ the excitation shown in Fig. 5a and with $K \leqq-0.1 I$ the excitation shown in Fig. $5 b$ 


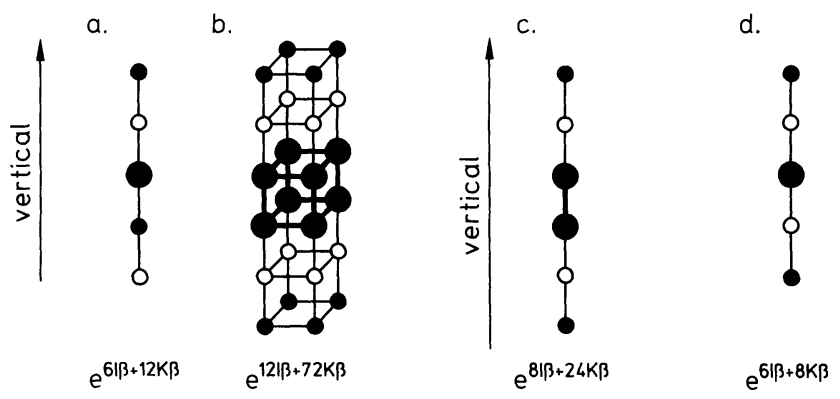

Fig. 5a-d. Excitations and their statistical weights near the line $I+4 J-2 K=0$

have the maximal statistical weight. In the corresponding main order $G_{1}=\min \{-6 I-12 K,-12 I-72 K\}$ as the occurrence frequency of these excitations is maximal for configurations $\langle 2,1\rangle$ equations $h(\langle 1\rangle)=h(\langle 2,1\rangle)$ and $h(\langle 2,1\rangle)=h(\langle 2\rangle)$ define curves $\mu_{1}(\beta)$ and $\mu_{2}(\beta)$ for which configurations $\langle 1\rangle$, $\langle 2,1\rangle,\langle 2\rangle$ are $G_{1}$-dominant ground states with $\mu \leqq \mu_{1}, \mu_{1} \leqq \mu \leqq \mu_{2}, \mu \geqq \mu_{2}$ respectively. With $K \leqq-0.1 I$ there is no other $G_{1}$-dominant ground state on the curve $\mu_{2}(\beta)$, but with $K \geqq-0.1 I$ all configurations consisting of layers of thickness 1 and 2 and having no adjacent layers of thickness 1 are $G_{1}$-dominant ground states. In the next orders this infinite degeneracy is removed by the excitation shown in Fig. $5 \mathrm{~b}$ with $-0.1 I \leqq K \leqq-(1 / 12) I$ and by the excitation shown in Fig. 5c with $K \geqq-(1 / 12) I$.

All layered configurations consisting of layers of thickness 1 or 2 and having no adjacent layers of thickness 2 are $G_{1}$-dominant ground states on the curve $\mu_{1}(\beta)$. This infinite degeneracy is removed in the order $-6 I-8 K$ by means of the excitation shown in Fig. 5d.

Remark. For the ANNNI model defined by the Hamiltonian:

$$
H\left(\varphi\left(\mathbb{Z}^{3}\right)\right)=-J_{0} \sum \varphi(x) \varphi(y)-J_{1} \sum \varphi(x) \varphi(y)+J_{2} \sum \varphi(x) \varphi(y),
$$

where the first summation goes over all horizontal nearest neighbours, the second summation goes over all vertical nearest neighbours, the third summation goes over all vertical next nearest neighbour's infinite family of curves $\delta=\delta_{n}(\beta)$ which

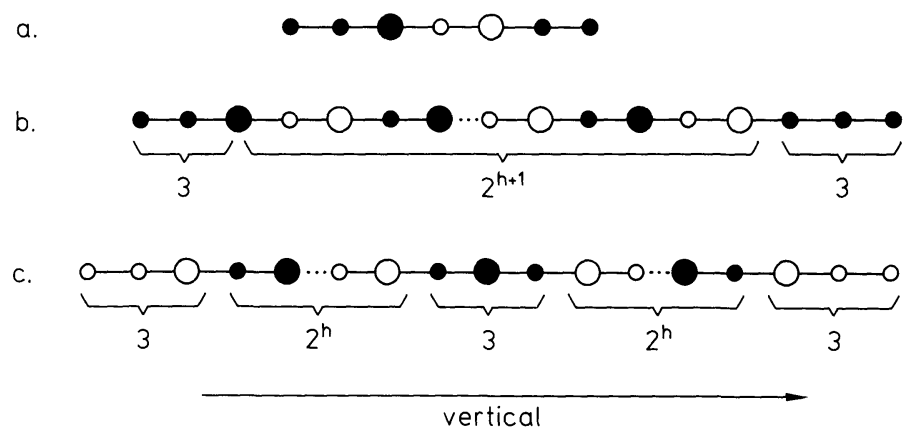

Fig. 6a-c. Excitations and its statistical weights for the ANNNI model 
phases $\left\langle 3,2^{n-1}\right\rangle$ (i.e. $\langle 3, \underbrace{2, \ldots, 2}_{n-1}\rangle$ ) and $\left\langle 3,2^{n-2}\right\rangle$ coexist on grows from point $\delta=0$ $\left(\delta=J_{1}-2 J_{2}\right)$. The difference between the ANNNI model and the microemulsion model in particular consists in distinction between excitations removing the infinite degeneracy on the phases $\langle 2\rangle$ and $\langle 3\rangle$ coexistence curve. This excitation for the microemulsion model was shown in Fig. $4 \mathrm{c}$ and for the ANNNI model it is shown in Fig. 6a. Moreover the phase $\left\langle 3,2^{n+1}\right\rangle$ appears between phases $\left\langle 3,2^{n}\right\rangle$ and $\langle 2\rangle$ because of the excitation illustrated in Fig. $6 \mathrm{~b}$, and $\left\langle 3,2^{n}\right\rangle-\left\langle 3,2^{n+1}\right\rangle$ boundary becomes stable because of the excitation illustrated in Fig. 6c. The last excitation defines the main order $G_{n+2}=(16 n+24) J_{0}+2 J_{1}$ which is used for construction of the curve $\delta_{n+2}(\beta)$. The smaller main order $G_{n+2}=(8 n+16) J_{0}$ and smaller excitation were erroneously indicated in [5].

Acknowledgements. We would like to thank Ya. G. Sinai for interest in the problem and S. B. Shlosman for useful remarks.

\section{References}

1. Widom, B.: The lattice microemulsion model. J. Chem. Phys. 84, 6943 (1986)

2. Dawson, K.A., Lipkin, M.D., Widom, B.: Phase diagram of a lattice microemulsion model. Preprint, Cornell University, USA 1987

3. Fisher, M.E., Selke, W.: Low-temperature analysis of the axial next nearest neighbour Ising model near its multiphase point. Phil. Trans. Soc. 302, 1 (1981)

4. Sinai, Ya.G.: Theory of phase transitions. Rigorous results. Budapest: Akademiai Kiado 1982

5. Dinaburg, E.I., Mazel', A.E., Sinai, Ya.G.: The ANNNI model and contour models with interaction. In: Mathematical physics reviews. Sov. Sci. Reviews Set C, Novikov, S.P. (ed.), vol. 6. New York: Gordon and Breach 1986

6. Bricmont, J., Slawny, J.: Phase transitions in systems with finite number of dominant ground states. Preprint Virginia Polytechnic Institute and State University, USA 1988

Communicated by Ya. G. Sinai

Received January 5, 1989 